\title{
IN VITRO ANTIHELMINTHIC ACTIVITY OF ETHANOL ZINGIBER OFFICINALE EXTRACT ON FASCIOLA GIGANTICA IN COMPARISON TO TRICLABENDAZOLE BY
}

\author{
FAWZIA H. TOULAH ${ }^{1}$, SAFINAZ J. ASHOOR ${ }^{1}$, MAJED H. WAKID ${ }^{2}$
} AND MONA R. ALSHATHLY ${ }^{*}$

Department of Biological Sciences, University of Jeddah ${ }^{1}$, Jeddah, Faculty of Applied Medical Sciences, King Abdul Aziz University ${ }^{2}$, Jeddah, Department of Biological Sciences, Northern Border University ${ }^{3}$, Arar, Saudi Arabia

( ${ }^{*}$ Correspondence: mrabdullah@uj.edu.sa)

\begin{abstract}
Fascioliasis, caused by Fasciola hepatica and F. gigantica, is considered one of the most important helminthes diseases among both humans and animals. The use of triclabendazole (TCBZ) as the only antihelminthic drug against fascioliasis faced recent problems being of many side effects and development of the drug resistance by the parasites. Given the widespread use of ginger (Zingiber officinale) in many traditional medicines and the various metabolic properties, this study aims to investigate the in vitro antihelminthic activity of $Z$. officinale ethanol extracts on $F$. gigantica in comparison to TCBZ. Fifty-four live adult $F$. gigantica worms were divided into nine groups of six in each, including positive control (G1), negative control (G2), triclabendazole sulfoxide (TCBZSO) of $20 \mu \mathrm{g} / \mathrm{ml}(\mathrm{G} 3)$, ginger extract of 5 , 25 and $50 \mathrm{mg} / \mathrm{ml}(\mathrm{G} 4, \mathrm{G} 5$ and G6, respectively) and combined TCBZSO with ginger extract of 5,25 and $50 \mathrm{mg} / \mathrm{ml}$ (G7, G8 and G9, respectively). The worm motility scores, survival index and histological examination were utilized to further analyze the effect of treatments on the worms' morphology. Results indicated a marked decrease in worms' motility treated with ginger extracts compared to TCBZSO group. The observed decrease was positively correlated to both time and concentration. Histological examination showed that a higher concentration of ginger extract alone or in combination with TCBZSO caused severe tegumental alterations, more than those observed in TCBZSO treatment alone. In conclusion, the results strongly confirm the plausible development of ginger-based antihelminthic drug against $F$. gigantica infection.
\end{abstract}

Keywords: Fasciola gigantica, Zingiber officinale, Antihelminthic activity, in vitro

\section{Introduction}

Fascioliasis is a major helminthes disease among humans and animals that inflect both serious health problems as well as significant economic losses worldwide (Alajmi, 2019). It is caused by two trematodes of the genus Fasciola; $F$. hepatica (temperate liver fluke) and $F$. gigantica (tropical liver fluke). The distribution of both species can overlap clinically in many regions of Africa and Asia (Degheidy and Al-Malki, 2012; Ashrafi et al, 2014; Phalee et al, 2015).

The fascioliasis effect on livestock is considered a significant problem as the infection usually leads to reduced growth, poor meat and milk production as well as other complications such as reduced fertility, abortion in late stages of pregnancy, anemia and even mortality, resulting in an annual economic loss of US $\$ 3$ billion (Jaja et al, 2017).

The World Health Organization (WHO) classifies fascioliasis as a neglected tropical disease, with an estimated 17 million people infected and 180 million people at risk of infection (Mas-Coma et al, 1999). The significant impact on agriculture and human health combined with increased demand for animal-derived food products to support global population growth suggested that fascioliasis is a major health problem (Cwiklinski et al, 2016).

The pathogenesis involved different phases of the fluke's life cycle inside the liver, which causes hepatitis, maturity and the establishment of the adult parasite in the bile ducts causing infection in the obstructive 
biliary tract. The main pathological changes in fascioliasis occur because of the immature migratory stage of the fluke. This stage of infection can cause extensive hemorrhage and fibrosis in the liver as the young flukes move through the liver. Adults in bile ducts cause inflammation and edema, which in turn stimulate fibrosis in the walls of bile ducts, resulting in atrophy of liver parenchyma, cirrhosis, and impaired liver function. In severe infections, the gall bladder is damaged, and the walls of bile ducts are completely eroded; therefore, the worms re-enter liver, again causing marked eosinophilic and granulomatous reaction (Raina et al, 2011; Nassef et al, 2014; Jaja et al, 2017).

In the last decades, animal fascioliasis outbreaks have spread to different parts of Saudi Arabia (Khanjari et al, 2014). Fasciola infection in sheep was considered a very serious problem in Riyadh Animal Markets, which forced consumers to shift from domestic to imported sheep (Degheidy and AlMalki, 2012).

There are no effective commercial vaccines yet; the anthelmintic drugs were mainly used to control the fluke (Massoud et al, 2012). Triclabendazole (TCBZ) is the drug of choice used for treating Fasciola infection. It can affect both stages of the fluke (juvenile and adult), providing effective control for both acute and chronic stages of fascioliasis. Various studies confirmed that the TCBZ-resistance in livestock worldwide (Alvarez-Sanchez et al, 2006; Ortiz et al, 2013; Shalaby et al, 2016). Such confirmations convey a serious issue, as there are no other effective drugs available (Massoud $e t$ al, 2008).

With respect to new compounds for use as antihelminthics, there has been increasing interest in natural plant products used as traditional medicines in developing countries (Flynn et al, 2010; Nassef et al., 2014).

One of the most famous herbs used as spice and for medicinal purposes is ginger (Zingiber officinale Roscoe). It belongs to the tropical and subtropical family Zingiber- aceae, originating in Southeast Asia (Gupta and Sharma, 2014). Ginger has been identified as an herbal medicinal product with pharmacological effect (Grzanna et al, 2005; Abouel-Nour et al, 2015). The ginger extract has been used since old times to treat diseases such as rheumatoid arthritis, sore throats, nausea, constipation and infectious diseases like helminthiasis (Gupta et al, 2016).

The use of $Z$. officinale to treat parasitic infections has received considerable attention lately. Several experimental and clinical trials have demonstrated ginger for its range of antihelminthics activity against schistosomiasis (Mostafa et al, 2011) and the protoscolices of hydatid cyst (Moazeni and Ahmadi, 2016). Thus, it has an antiprotozoal effect against Toxoplasma gondii (Choi et al, 2013), Giardia lamblia (Mahmoud et al, 2014), Trypanosoma brucei brucei and Blastocystis spp. (El-Sayed and El-Saka, 2015). Besides, Moazeni and Khademolhoseini (2016) showed that $F$. hepatica eggs were susceptible to methanolic extract of ginger with ovicidal effect of $100 \%$ after 24 and 48 hours at concentrations of 10 and $5 \mathrm{mg} / \mathrm{ml}$, respectively.

The effect of $Z$. officinale on $F$. gigantica has not been studied previously; therefore, the current study aimed to investigate the in vitro antihelminthic activity of $Z$. officinale ethanol extract on the $F$. gigantica in comparison to TCBZ.

\section{Materials and Methods}

Liver flukes: Fifty-four live $F$. gigantica adult worms were collected from the bile ducts of the naturally infected cattle and sheep slaughtered at Jeddah slaughterhouse. To eliminate all traces of blood and bile, the flukes were washed several times with warm normal saline solution $\left(37^{\circ} \mathrm{C}\right)$ and the healthy flukes with normal histological structure and good motility were selected. Healthy adult flukes were kept in a petri dish containing 20ml of M-199 medium (SigmaAldrich, St. Louis, MO, USA) and transferred to the lab of Parasitology Department, Jeddah University, Saudi Arabia, until the 
experimental beginning.

Plant extract: $Z$. officinale rhizomes were purchased from local market in Jeddah, Saudi Arabia. The rhizomes were cleaned, scrapped of superficial skin, cut into small pieces, air-dried for a week then ground using a mechanical grinder. The ground product was macerated in absolute ethanol for 24 hours, filtered with a clean white cloth and the filtrate was concentrated using a rotary evaporator in a water bath at $40-50{ }^{\circ} \mathrm{C}$. The ethanolic extract was refrigerated until used (Moazeni and Khademolhoseini, 2016). For final use, the ethanolic residue was dissolved in M-199 culture medium immediately before use. Three doses of ginger ethanolic extract were investigated, $5,25 \& 50 \mathrm{mg} / \mathrm{ml}$ (Bazh and El-Bahy, 2013).

Drug and media: Triclabendazole (SigmaAldrich) was initially prepared as a stock solution in dimethyl sulfoxide (DMSO) and then added to the culture medium M-199 (Sigma-Aldrich) containing antibiotics (penicillin $50 \mathrm{IU} / \mathrm{ml}$ and gentamycin $30 \mathrm{IU} / \mathrm{ml}$ ) to give a maximum concentration of $0.1 \%$ (V/V) (Mestorino et al, 2008). A concentration of $20 \mu \mathrm{g} / \mathrm{ml}$ triclabendazole sulfoxide (TCBZSO) was used (Sanyal, 1995).

Experimental design: Adults $F$. gigantica were recovered under sterile conditions in a laminar flow cabinet. The worms were kept in petri dish containing $20 \mathrm{ml} \mathrm{M}-199$ medium. They were divided into nine groups containing six flukes each. Group (1) included six non-drug exposed $F$. gigantica worms served as positive control group and were incubated in M-199 medium (Chang and Flors, 2015). Group (2) included six $F$. gigantica worms incubated in M-199 medium containing $0.1 \%$ DMSO served as negative control group (Shalaby et al, 2016). Group (3) included six $F$. gigantica worms incubated in M-199 medium containing 20 $\mu \mathrm{g} / \mathrm{ml}$ of TCBZSO (Hegazy et al, 2007). Groups $(4,5 \& 6)$ each included six $F$. gigantica worms exposed to $5,25 \& 50 \mathrm{mg} / \mathrm{ml}$ ginger extract, respectively (Bazh and ElBahy, 2013). Groups (7, 8 \& 9) included six
$F$. gigantica worms each, exposed to $5,25 \&$ $50 \mathrm{mg} / \mathrm{ml}$ ginger extract with the $20 \mu \mathrm{g} / \mathrm{ml}$ TCBZSO, respectively.

Parasitic viability: Worms' viability was evaluated in each group using a stereomicroscope (Chang and Flors, 2015). The inhibition of motility and/or mortality of flukes were observed after 3, 6, $12 \& 24 \mathrm{hrs}$.

Parasitic survival index: Survival index (SI) was evaluated using stereomicroscope. Percentages of live worms after a given time (3, 6,12 or $24 \mathrm{hrs})$ were evaluated with scored system (Jiraungkoorskul et al, 2005).

Morphological identification of adult Fasciola: One fluke from each group was fixed in $10 \%$ buffered formalin, dehydrated, cleared and embedded in paraffin. Serial $5 \mu \mathrm{m}$ sections were stained with hematoxylin and eosin (H\&E) after Bancroft and Gamble (2008) for routine histological study. The layers of the tegument were examined under light microscope to check for abnormalities and get photographed (Hanna et al, 2013).

Statistical analysis Correlation data between TCBZ treatment and ginger treatment were entered into Microsoft excel sheet and were analyzed using IBM Corp. IBM SPSS Statistics for Windows, Version 22.0. (Armonk, NY, United States). Further comparison using Chi-square was applied and significance appointed at $\mathrm{P}$ value of $<0.5$.

\section{Results}

The motility/mortality scores of all treated groups were observed under a stereomicroscope with scores ranging between (score 3 ) for normal whole-body movement to (score 0 ) for dead. All treated groups were observed after 3, 6 or $12 \mathrm{hrs}$ (Tab. 1). The positive control (G1 group) and negative control (G2 group) showed no change in worms' motility (score 3) after all incubation times points. The group treated with $20 \mu \mathrm{g} / \mathrm{ml}$ of TCBZSO (G3) showed reduced movement (score 2) for most of the flukes after $3 \mathrm{hrs}$ of incubation and the motility score decreased after 6 hours to include three dead (score 0), two immobilized and alive (score 1) and one reduced movement (score 2). After 12hrs of 
incubation, most of them were dead (score $0)$.

But, the ginger treated groups (G4, G5 \& G6) with increased ginger extract dose (5, $25,50 \mathrm{mg} / \mathrm{ml}$, respectively) showed concentration- and time-dependent changes. Group 4 showed only two flukes with reduced movement (score 2) after $3 \mathrm{hrs}$ of incubation while after 6hrs, there was one dead (score 0 ), one immobilized and alive (score 1) and two with reduced movement. After $12 \mathrm{hrs}$, most of the flukes were dead (score 0) with one immobilized and alive (score 1). The effect of increased dose in G5 was markedly observed after 3 hours of incubation as there were two dead flukes (score 0), three immobilized and alive (score 1) and one with reduced movement, while after $6 \mathrm{hrs}$ the number increased to four dead (score 0) and two immobilized (score 1). All flukes were dead after $12 \mathrm{hrs}$ of incubation. Similar correlations were observed in G6 as most of the flukes died (score 0 ) after $3 \mathrm{hrs}$ of incubation and all flukes were dead after 6 and 12hrs.

The combined ginger and TCBZSO treated groups (G7, G8 \& G9) with $20 \mu \mathrm{g} / \mathrm{ml}$ of TCBZSO and increased ginger extract dose $(5,25,50 \mathrm{mg} / \mathrm{ml}$, respectively) had the most intense effect on flukes regardless of the incubation time. Three hours incubation revealed two dead (score 0) in G7, five in G8 and all six in G9, while after 6hrs the dead flukes increased to four in $\mathrm{G} 7$ and all six in G8 and G9. The 12hrs incubation showed all six flukes dead in G7, G8 \& G9.

Statistical analyses were applied on data and showed Chi-square value of 221.833 with significant $P$ values $(<0.05)$.

Survival index scores (in percentage) of $F$. gigantica after $3,6,12 \& 24$ hours were recorded under stereomicroscope. The control groups (G1and G2) exhibited active movement and maximum survival index (SI = $100 \%$ ) after all incubation times. The TCBZSO treated group (G3) showed declining SI (from $100 \%$ to $16.6 \%$ ) with increasing incubation time. Similar decline was observed in both the ginger treated groups (G4,
G5 \& G6) and combined ginger and the TCBZSO treated groups (G7, G8 \& G9) in accordance with time and concentration increase (Tab. 2, Fig. 1).

Control groups (G1 \& G2) showed no abnormalities and normal histological composition of the tegument, which was lodged with spins enclosed with the muscle layer and parenchyma. The outer rim of the tegument and tips of the spines were deeply stained. The tegument rested on basement membrane underlying muscle layers (longitudinal and diagonal) surrounding the mesenchyma (Fig. 2).

Group 3, treated with TCBZSO, showed mild separation of tegument between the spines from underlying tissue with dislodged spines (Fig. 3).

The sever histological changes were observed in the ginger extract treated groups (G4, G5 \& G6) as well as a positive correlation with extract concentration. Group 4 showed swelling intact tegument, embedded spines and distracted muscle layers (Fig. 4A) while group 5 showed severe swelling of the tegument, partial separation between the tegument and the distracted muscle layers underneath with the spines either surrounded by the tegument or completely dislodged (Figure 4B). Moreover, group 6 showed separated tegument and marked multiple dislodged spines with furrows embedded into the muscle layer (Fig. 4C).

The combined ginger and TCBZSO treated groups (G7, G8 \& G9) had the most sever observations compared to all other groups. Group 7 showed swelled and detached tegument, dislodged spines with empty spine sockets and extensive cracking of the muscle layer (Fig. 5A). In addition to G7 observations, G8 also showed clearly surrounded spines by tegument with some embedded into the muscle layer as well as the appearance of many vacuoles (Fig, 5B). Group 9 showed completely detached tegument with appearance of multiple empty spine sockets and sever destruction of the underlying muscle layer (Fig.5C). 
Table 1: Motility scores count of $F$. gigantica worms' number after $3,6 \& 12$ hrs incubation (each n=6 worms).

\begin{tabular}{|c|c|c|c|c|c|}
\hline \multicolumn{2}{|c|}{ Variables } & \multicolumn{4}{|c|}{ Motility Scores } \\
\hline Group & Time (hrs) & Dead (0) & Immobile $\&$ alive (1) & Reduced movement (2) & Normal movement (3) \\
\hline \multirow{3}{*}{ G 1} & 3 & 0 & 0 & 0 & 6 \\
\hline & 6 & 0 & 0 & 0 & 6 \\
\hline & 12 & 0 & 0 & 0 & 6 \\
\hline \multirow{3}{*}{ G 2} & 3 & 0 & 0 & 0 & 6 \\
\hline & 6 & 0 & 0 & 0 & 6 \\
\hline & 12 & 0 & 0 & 0 & 6 \\
\hline \multirow{3}{*}{ G 3} & 3 & 0 & 0 & 5 & 1 \\
\hline & 6 & 3 & 2 & 1 & 0 \\
\hline & 12 & 5 & 1 & 0 & 0 \\
\hline \multirow{3}{*}{ G 4} & 3 & 0 & 0 & 2 & 4 \\
\hline & 6 & 1 & 1 & 2 & 2 \\
\hline & 12 & 4 & 1 & 1 & 0 \\
\hline \multirow{3}{*}{ G 5} & 3 & 2 & 3 & 1 & 0 \\
\hline & 6 & 4 & 2 & 0 & 0 \\
\hline & 12 & 6 & 0 & 0 & 0 \\
\hline \multirow{3}{*}{ G 6} & 3 & 5 & 1 & 0 & 0 \\
\hline & 6 & 6 & 0 & 0 & 0 \\
\hline & 12 & 6 & 0 & 0 & 0 \\
\hline \multirow{3}{*}{ G 7} & 3 & 2 & 0 & 2 & 2 \\
\hline & 6 & 4 & 1 & 1 & 0 \\
\hline & 12 & 6 & 0 & 0 & 0 \\
\hline \multirow{3}{*}{ G 8} & 3 & 5 & 1 & 0 & 0 \\
\hline & 6 & 6 & 0 & 0 & 0 \\
\hline & 12 & 6 & 0 & 0 & 0 \\
\hline \multirow{3}{*}{ G 9} & 3 & 6 & 0 & 0 & 0 \\
\hline & 6 & 6 & 0 & 0 & 0 \\
\hline & 12 & 6 & 0 & 0 & 0 \\
\hline
\end{tabular}

Table 2: Survival index (SI) values (\%) of $F$. gigantica worms after different incubation times.

\begin{tabular}{|c|c|c|c|}
\hline Variables & \multicolumn{3}{|c|}{ Incubation Time } \\
\hline Group & 3 hours & 6 hours & 12 hours \\
\hline G 1 & 100 & 100 & 100 \\
\hline G 2 & 100 & 100 & 100 \\
\hline G 3 & 100 & 50 & 16.6 \\
\hline G 4 & 100 & 83.3 & 33.3 \\
\hline G 5 & 66.6 & 33.3 & 0 \\
\hline G6 & 16.6 & 0 & 0 \\
\hline G 7 & 66.6 & 33.3 & 0 \\
\hline G 8 & 16.6 & 0 & 0 \\
\hline G 9 & 0 & 0 & 0 \\
\hline
\end{tabular}

\section{Discussion}

Fascioliasis is a foodborne zoonotic disease caused by the two parasite species Fasciola hepatica and Fasciola gigantica (Grabner et al, 2014). The effectiveness of using natural plant extracts as alternative drugs has been heavily investigated in recent years (Anthony et al, 2012; Nassef et al, 2014); especially in cases were the drug can cause deleterious side effects and/or have acquired resistance (Hanna et al, 2015; Kel- ley et al, 2016), as antihelminthics drugs controlling fascioliasis (Geary et al, 2012; Hossain et al, 2012).

Ginger has been reported in many studies to cause marked the significant nematocidal, cestocidal, trematocidal, antiprotozoal, insecticidal, molluscicidal and anti-leech effects (Al-Sharkawi et al, 2007; Choi et al, 2013; Lin et al, 2014; El-Sayed and El-Saka, 2015; Abouel-Nour et al, 2016; Dyab et al, 2016, Fakhrieh-Kashan et al, 2018). Moreover, 
Iqbal et al. (2006) reported that the ginger anthelmintic activity against gastrointestinal nematodes in sheep. Also, Abdel-Hafeez et al. (2015) proved the in vivo antiprotozoan effects of the Zingiber officinale extracts on experimentally infected mice with Blastocystis hominis.

For such reasons, this study pioneered the in vitro antihelminthic activity of $Z$. officinale (ginger) ethanol extracts on $F$. gigantica in comparison to the commonly used TCBZ drug.

In the present study, ginger ethanolic extract have demonstrated strong fasciolicidal activity independently and in combination with TCBZSO drug with the fluke's tegument being a major target. The tegument isolates the fluke from the surrounding environment, maintains its homeostasis, absorbs nutrients, and defends direct contact with drugs, in addition to synthesis and secretions of antigens (Anuracpreeda et al, 2017). So, damage of tegument cause vital changes that can affect many downstream internal processes.

The effect on fluke's tegument was markedly observed both in motility/mortality scores as well as in histological examination of the worm, which is an important parameter for estimating the effectiveness of any anthelmintic drugs (Shalaby et al, 2009). All treated groups were observed after 3,6 or $12 \mathrm{hrs}$ and the results were positively correlated with both time and concentration. The positive and negative control groups (G1 \& G2, respectively) showed normal composition of the tegument, spines, muscles and basement membrane after all tested incubation times. Similar results were confirmed with others (Anuracpreeda et al, 2017). These flukes groups showed normal active movement (score 3) and maximum SI $(100 \%)$. These results agreed with Jeyathilakan et al. (2010) and Ullah et al. (2017) who reported that all control liver flukes remain active and motile after different time of incubation.

The group treated with $20 \mu \mathrm{g} / \mathrm{mL}$ TCBZSO
(G3) showed reversed correlation between motility and time with higher mortality scores after 6 hours of incubation. This could be attributed to the inhibited secretion of proteolytic enzyme and disrupted microtubulebased secretory processes that responded to the worm mobility (Massoud et al, 2012). Histological examination showed mild separation between the spines from underlying tissue with dislodged spines, which concur with previously published findings of disrupted tegument, spine loss and decreased worms' motility after 24 hours incubation with $15 \mu \mathrm{g} / \mathrm{mL}$ TCBZSO (Massoud et al, 2012). Besides, Shalaby et al. (2009) and McConville et al. (2009) reported that disruption and spine loss were characteristic of a stressed fluke.

In the present study, the groups treated with different concentrations of ginger extract $(5,25 \& 50 \mathrm{mg} / \mathrm{ml}$; G4, G5 \&G6, respectively) revealed sever and significant decrease in the worms motility as well as increased mortality scores in positive correlation with both time and concentration. Histological examination showed disrupted tegument, in the form of mild swelling with partial separation in low ginger concentration (G4) and marked swelling with multiple sloughed spines and embedded furrows in the muscle layer in high ginger concentrations (G5 \& G6). These tegumental changes immobilized the flukes then died. Similar observations were confirmed by Jeyathilakan et al. (2010); Shalaby et al. (2016) and Anuracpreeda et al. (2017) who demonstrated that the tegumental swelling is the first sign of change and characterized by osmotic disturbance due to the destruction of the tegument membrane and plasma membranes' ion pumps. This swelling of tegument is the main target of many flukicides pharmacological action (Devine et al, 2012).

In the present study, TCBZSO group (G3) and ginger extract groups (G4, G5 \& G6) indicated that higher ginger dosage afflict more sever observations than the drug. Such observation confirmed the effectiveness of 
the ginger extracts as an alternative drug to treat fascioliasis infection.

The combination of the $20 \mu \mathrm{g} / \mathrm{ml}$ TCBZSO and different ginger extract concentrations $(5,25 \& 50 \mathrm{mg} / \mathrm{ml}$; G7, G8 \& G9, respectively) had a foreseeable sever and stringent effect in as early as post-3 hours of incubation. Low motility and high mortality scores were markedly observed as well as very low SI scores. These results confirm previous reports that $Z$. officinale extract markedly reduced the motility and caused death of flukes (score 0) after 3 hours (Jeyathilakan et al, 2010). In addition, histological examination showed severe dislodgment of many spines from their sockets, the muscle layer appeared markedly cracking with many vacuoles and the tegument had been completely dislocated in some specimen. Shalaby et al. (2016) reported that the tegument once detached, the drug would be able to penetrate deeper into the internal tissues of the fluke leading to a more widespread destruction. Such tegumental damage may trigger the release of the surface antigen that interacts with specific antibodies in the blood, promoting a rapid and severe immunopathological response to destruct and kill the fluke.

\section{Conclusion}

The outcome results strongly support a plausible development of ginger-based antihelminthics drug against $F$. gigantica infection. The combination of ginger extract $(5$, $25 \& 50 \mathrm{mg} / \mathrm{ml}$ ) and $20 \mu \mathrm{g} / \mathrm{ml}$ the TCBZSO showed fasciolicidal properties of the $Z$. officinale.

These compounds inhibited the worms' motility and severely disrupt the tegumental surface. Furthermore, molecular, ultrastructural, and physiological studies are recommended to evaluate ginger extract effect.

\section{References}

Abdel-Hafeez, EH, Ahmad, AK, Kamal, AM, Abdellatif, MZ, Abdelgelil, NH, 2015: In vivo antiprotozoan effects of garlic (Allium sativum) and ginger (Zingiber officinale) extracts on experimentally infected mice with Blastocystis spp. Parasitol. Res. 114, 9:3439-44

Abouel-Nour, MF, El-Shewehy, DMM, Ham- ada, SF, Morsy, TA, 2015: The efficacy of three medicinal plants: garlic, ginger and mirazid and a chemical drug metronidazole against Cryptosporidium parvum. I- Immunological response. J. Egypt. Soc. Parasitol.. 45, 3:559-70.

Abouel-Nour, MF, El-Shewehy, DMM, Hamada, SF, Morsy, TA, 2016: The efficacy of three medicinal plants; garlic, ginger and mirazid and a chemical drug metronidazole against Cryptosporidium parvum: II- Histological changes. J.

Egypt. Soc. Parasitol. 46, 1:185-200.

Ahmad, MM, El Hady, HM, Morsy, TA, 1990: Parasitic infections and haemoglobin level among school children of different socio-economic classes in Abha, Saudi Arabia. J. Egypt. Soc. Parasitol. 20, 1:61-8.

Alajmi, RA, 2017: Molecular characterization of Fasciola flukes using mitochondrial28S rRNA gene in Naimi Saudi sheep. Saudi J. Biol. Sci. 26, 1:112-117.

Al-Sharkawi, IM, El-Shaikh, KA, Tabl, GA, Ali, JA, 2007: Effect of ginger on Schistosoma mansoni infected mice. Delta J. Sci. 31:1-10.

Alvarez-Sanchez, M, Mainar-Jaime, R, PerezGarcia, J, Rojo-Vázquez, F, 2006: Resistance of Fasciola hepatica to triclabendazole and albendazole in sheep in Spain. Vet. Record 159, 13: 424-8.

Amniyom, A, Panyarachun, B, Puttarak, P, et al, 2017: The in vitro anthelmintic activity of the ethanol leaf extracts of Terminalia catappa $\mathrm{L}$. on Fasciola gigantica. Parasitology 144, 14: 193142.

Anthony, MP, Burrows, JN, Duparc, S, Moehrle, J, Wells, TN, 2012: The global pipeline of new medicines for the control and elimination of malaria. Malar. J. 11, 1:316-20.

Anuracpreeda, P, Chawengkirtikul, R, Ng-Ba zh, EK, El-Bahy, NM, 2013: In vitro and in vivo screening of anthelmintic activity of ginger and curcumin on Ascaridia galli. Parasitol. Res. 112, 11:3679-86.

Ashrafi, K, Bargues, MD, O'Neill, S, Mas-Coma, S, 2014: Fascioliasis: a worldwide parasitic disease of importance in travel medicine. Travel Med. Infect. Dis. 12, 6:636-49.

Chang, ACG, Flores, MJC, 2015: Morphology and viability of adult Fasciola gigantica (giant liver flukes) from Philippine carabaos (Bubalus bubalis) upon in vitro exposure to lead. Asian Pac. J. Trop. Biomed. 5, 6:493-6.

Choi, WH, Jiang, MH, Chu, JP, 2013: Antiparasitic effects of Zingiber officinale (ginger) 
extract against Toxoplasma gondii. J. Appl. Biomed. 11:15-26.

Cwiklinski, K, O'Neill, SM, Donnelly, S, Dalton, JP, 2016: A prospective view of animal and human fasciolosis. Parasit. Immunol. 38, 9:55868.

Degheidy, NS, Al-Malki, JS, 2012: Epidemiological studies of fasciolosis in human and animals at Taif, Saudi Arabia. World Appl. Sci. J, 19:1099-104.

Devine, C, Brennan, GP, Lanusse, CE, Alvarez, LI, Trudgett, A, et al, 2012: Potentiation of triclabendazole action in vivo against a triclabendazole-resistant isolate of Fasciola hepatica following its co-administration with the metabolic inhibitor, ketoconazole. Vet. Parasitol. 184, 1:37-47.

Dyab, AK, Yones, DA, Ibraheim, ZZ, Hassan, TM, 2016: Anti-giardial therapeutic potential of dichloromethane extracts of Zingiber officinale and Curcuma longa in vitro and in vivo. Parasitol. Res. 115, 7:2637-45

El-Sayed, NM, El-Sakla, MM, 2015: Anti-parasitic activity of Zingiber officinale (ginger): A brief review. Aperito J. Bact. Virol, Parasitol. 2: 1-6.

Fakhrieh-Kashan, Z, Arbabi, M, Delavari, M, Mohebali, M, Hooshyar, H, 2018: Induction of apoptosis by alcoholic extract of combination of Verbascum Thapsus and Ginger officinale on Iranian isolate of Trichomonas vaginalis. Iran. J. Parasitol. 13, 1:72-8.

Flynn, RJ, Mulcahy, G, Elsheikha, HM, 2010: Coordinating innate and adaptive immunity in Fasciola hepatica infection: implications for co-ntrol. Vet. Parasitol. 169, 3/4: 235-40.

Garcia, LS, 2001: Diagnostic Medical Parasitology. $4^{\text {th }}$ ed.: ASM Press, Washington, DC.

Geary, TG, Chibale, K, Abegaz, B, AndraeMarobela, K, Ubalijoro, E, 2012: A new approach for anthelmintic discovery for humans. Trends Parasitol. 28, 5:176-81.

Grabner, DS, Mohamed, FA, Nachev, M, Méabed, EM, Sabry, AH, et al, 2014: Invasion biology meets parasitology: a case study of parasite spill-back with Egyptian Fasciola gigantica in the invasive snail Pseudosuccinea columela. PLoS One Feb 11; 9, 2:e88537. doi: 10.1371/ journal.pone.0088537.

Grzanna, R, Lindmark, L, Frondoza, CG, 2005: Ginger: An herbal medicinal product with broad anti-inflammatory actions. J. Med. Food, $8,2: 125-32$.
Gupta, R, Singh, PK, Singh, R, Singh, RL, 2016: Pharmacological activities of Zingiber officenale (ginger) and its active ingredients: A review. Inter. J. Sci. Innov. Res. 4:1-18.

Gupta, SK, Sharma, A, 2014: Medicinal properties of Zingiber officinale Roscoe: A re-view. J. Pharm. Biol. Sci. 9, 5:124-9.

Hanna, REB, Forster, FI, Brennan, GP, Fairweather, I, 2013: Fasciola hepatica: Histological demonstration of apoptosis in the reproductive organs of flukes of triclabendazole-sensitive and triclabendazole-resistant isolates, and in field-derived flukes from triclabendazoletreated hosts, using in situ hybridisation to visualise endonuclease-generated DNA strand breaks. Vet. Parasitol. 191, 3/4:240-51.

Hanna, REB, McMahon, C, Ellison, S, Edgar, HW, Kajugu, PE, et al, 2015: Fasciola hepati$c a$ : A comparative survey of adult fluke resistance to triclabendazole, nitroxynil and closantel on selected upland and lowland sheep farms in Northern Ireland using fecal egg counting, coproantigen ELISA testing and fluke histology. Vet. Parasitol. 207, 1/2:34-43.

Hegazi, AG, El Hady, FKA, Shalaby, HA, 2007: An in vitro effect of propolis on adult worms of Fasciola gigantica. Vet. Parasitol. 144, 3/4:279-86.

Hossain, E, Chandra, G, Nandy, AP, Mandal, SC, Gupta, JK, 2012: Anthelmintic effect of a methanol extract of Bombax malabaricum leaves on Paramphistomum explanatum. Vet. Parasitol. 110, 3:1097-102.

Iqbal, Z, Lateef, M, Akhtar, MS, Ghayur, M N, Gilani, AH, 2006: In vivo anthelmintic activity of ginger against gastrointestinal ne-matodes of sheep. J. Ethnopharmacol. 106, 2: 285-7.

Jaja, IF, Mushonga, B, Green, E, Muchenje, V, 2017: Financial loss estimation of bovine fasciolosis in slaughtered cattle in South Africa. Parasit. Epidemiol. Control 2, 4:27-34.

Jeyathilakan, N, Murali, K, Anandaraj, A, Abdul Basith, S, 2010: In vitro evaluation of anthelmintic property of herbal plants against Fasciola gigantica. Indian J. Anim. Sci. 80, 11: 1070-4.

Jiraungkoorskul, W, Sahaphong, S, Tansatit, T, Kangwanragsan, N, Pipatshukiat, S, 2005: Eurytrema pancreaticum: The in vitro effect of praziquantel and triclabendazole on the adult fluke. Exp. Parasitol. 111, 3:172-7.

Kelley, JM, Elliott, TP, Beddoe, T, Anderson, G, Skuce, P, et al, 2016: Current threat of tri- 
clabendazole resistance in Fasciola hepatica. Trends Parasitol. 32, 6:458-69.

Khanjari, A, Bahonar, A, Fallah, S, Bagheri, M, Alizadeh, A, et al, 2014: Prevalence of fasciolosis and dicrocoeliosis in slaughtered sheep and goats in Amol Abattoir, Mazandaran, northern Iran. Asian Pac. J. Trop. Dis. 4, 2:120-4.

Mahmoud, A, Attia, R, Safaa, SAID, Ibraheim, Z, 2014: Ginger and cinnamon: Can this house-hold remedy treat giardiasis? Parasitological and histopathological studies. Iranian J. Parasitol. 9, 4:530-4.

Mas-Coma, MS, Esteban, JG, Bargues, MD, 1999: Epidemiology of human fascioliasis: A review and proposed new classification. Bull. WHO, 77, 4:340-6.

Massoud, AM, El-Shazly, AM, Awad, SE, Morsy, ATA, Sadek, GS, et al, 2006: New trends in diagnosis and treatment of chronic intestinal strongyloidiasis stercoralis in Egyptian patients. J. Egypt. Soc. Parasitol. 36, 3:827-44.

Massoud, AMA, Hafez, AO, Abdel-Gawad, E A, El Shazly, AM, Morsy, TA, 2008: Mirazid alone or combined with Paromomycin in treating cryptosporidiosis parvum in immunocompetent hospitalized patients J. Egypt. Soc. Parasitol., 2:399-418.

Massoud, AM, Shalaby, HA, El Khateeb, R M, Mahmoud, MS, Kutkat, MA, 2012: Effects of Mirazid ${ }^{\circledR}$ and myrrh volatile oil on adult Fasciola gigantica under laboratory conditions. Asian Pac. J. Trop. Biomed. 2, 11: 875-84.

McConville, M, Brennan, GP, Flanagan, A, Hanna, REB, Edgar, HWJ, et al, 2009: Surface changes in adult Fasciola hepatica following treatment in vivo with the experimental fasciolicide, compound alpha. Parasitol. Res. 105, 3: 757-60.

Mestorino, N, Formentini, EA, Lucas, MF, Fernandez, C, Modamio, P, et al, 2008: Pharmacokinetic disposition of triclabendazole in cattle and sheep; discrimination of the order and the rate of the absorption process of its active metabolite triclabendazole sulfoxide. Vet. Res. Commun. 32, 1:21-33.

Moazeni, M, Ahmadi, A, 2016: Controversial aspects of the life cycle of Fasciola hepatica. Exp. Parasitol. 169:81-9.
Moazeni, M, Khademolhoseini, AA, 2016: Ovicidal effect of the methanolic extract of ginger (Zingiber officinale) on Fasciola hepatica eggs: an in vitro study. J. Parasit. Dis.: Indian Society for Parasitology 40, 3:662-6.

Mostafa, OM, Eid, RA, Adly, MA, 2011: Antischistosomal activity of ginger (Zingiber officinale) against Schistosoma mansoni harbored in C57 mice. Parasitol. Res. 109, 2:395-403.

Nassef, NE, El-Kersh, WM, El Sobky, MM, Harba, NM, Khalil, SAER, 2014: In-vitro and in-vivo assessment of the effect of soybean extract on Fasciola gigantica infection in comparison with triclabendazole. Menoufia Med. J. 27, 1:93-8.

Ortiz, P, Scarcella, S, Cerna, C, Rosales, C, Cabrera, M, et al, 2013: Resistance of Fasciola hepatica against triclabendazole in cattle in Cajamarca (Peru): a clinical trial and an in vivo efficacy test in sheep. Vet. Parasitol. 195, 1/2:11821.

Phalee, A, Wongsawad, C, Rojanapaibul, A, Chai, JY, 2015: Experimental life history and biological characteristics of Fasciola gigantica (Digenea: Fasciolidae). Korean J. Parasitol. 53, 1:59-64.

Raina, OK, Nagar, G, Varghese, A, Prajitha, G, Alex, A, et al, 2011: Lack of protective efficacy in buffaloes vaccinated with Fasciola gigantica leucine aminopeptidase and peroxiredoxin recombinant proteins. Acta Tr-op.118, 3:217-22. Sanyal, PK, 1995: Kinetic disposition of triclabendazole in buffalo compared to cattle. J. Vet. Pharmacol. Therap. 18, 5:370-4.

Shalaby, HA, El Namaky, AH, Kamel, RO, 2009: In vitro effect of artemether and triclabendazole on adult Fasciola gigantica. Vet. Parasitol. 160, 1/2:76-82.

Shalaby, HA, El Namaky, AH, Kamel, RO, 2016: In vitro tegumental alterations on adult Fasciola gigantica caused by mefloquine. J. Parasit. Dis.: Indian Society for Parasitology 40, 1: 14-51.

Ullah, R, Rehman, A, Zafeer, MF, Rehman, L, Khan, YA, et al, 2017: Anthelmintic potential of thymoquinone and curcumin on Fasciola gigantica. PloS one, 12, 2: e0171267. doi: 10. 1371/ journal.pone. 0171267

\section{Explanation of figures}

Fig. 1: Bar diagram indicating survival index (SI) percentage of Fasciola gigantica worms after different incubation times.

Fig. 2: Light micrographs of adult $F$. gigantica worm stained with H\&E with normal histology. A: positive control (G1) showing tegument (T) with normal appearances, i.e., spines (S) embedded in tegument and muscle (M) lying underneath parenchyma (P). B: negative control (G2) in M-199 medium containing 0.1\% DMSO showed intact tegument (T) with lodged spines (S) enclosed with muscle layer (M) and parenchyma $(\mathrm{P})$. 
Fig. 3: Light micrographs of adult $F$. gigantica worm stained with H\&E and treated with 20 $\mathrm{\mu g} / \mathrm{ml}$ TCBZSO (G3) showed mild separation of tegument between the spines (S) and underlying tissue (T) and dislodged spines (DS).

Fig. 4: Light micrographs of adult $F$. gigantica worm stained with $\mathrm{H} \& \mathrm{E}$ and treated with $5,25 \mathrm{or} 50 \mathrm{mg} / \mathrm{ml}$ ginger ethanolic extract (G4, G5 \& G6, respectively). A: G4 showed swelled intact tegument (ST), embedded spines (ES) and distracted muscle layer (DM). B: G5 showed swelling and partial separation of tegument (ST) from a distracted muscle layer and spines (S) surrounded by tegument (ES) while others dislodged (DS). C, G6 showed marked multiple dislodged spines (DS), tegumental swelling with embedded spines (S) and detached with furrows through muscle layer (DT).

Fig. 5 Light micrographs of adult $F$. gigantica worm stained with H\&E and treated with 20ug/ml TCBZSO and 5, 25 or $50 \mathrm{mg} / \mathrm{ml}$ ginger ethanolic extract (G7, G8 \& G9, respectively). A, G7 showed swelled detached tegument (ST), dislodged spines (DS), empty spine sockets (arrow) and extensive cracking of the muscle layer (M). B, G8 showed gross tegumental swelling (ST), spines surrounded by tegument and embedded in the muscle layer (EM) with appearance of many vacuoles (*). C, G9 showed complete detached tegument (DT) with multiple empty spine sockets (arrows).
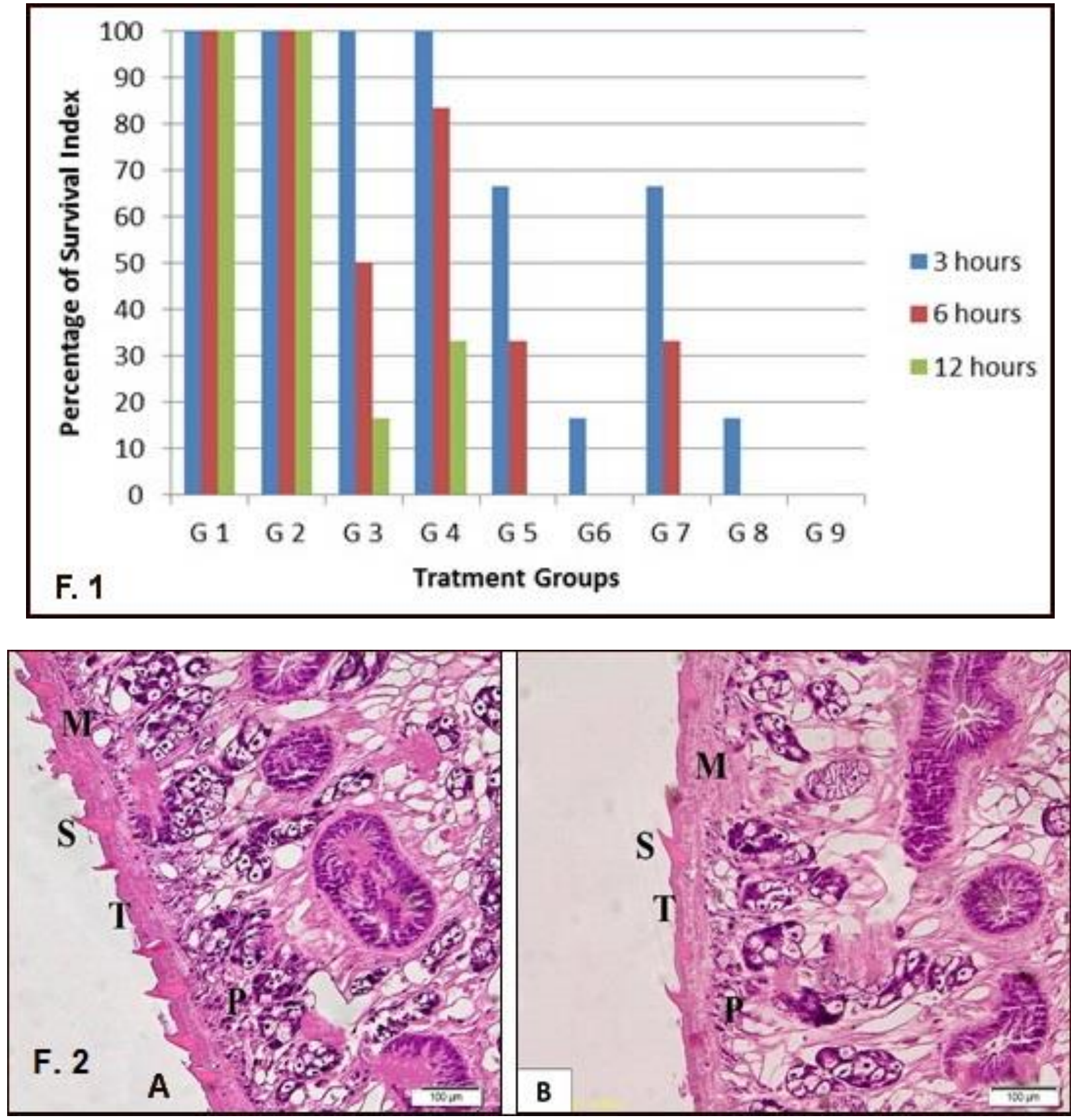

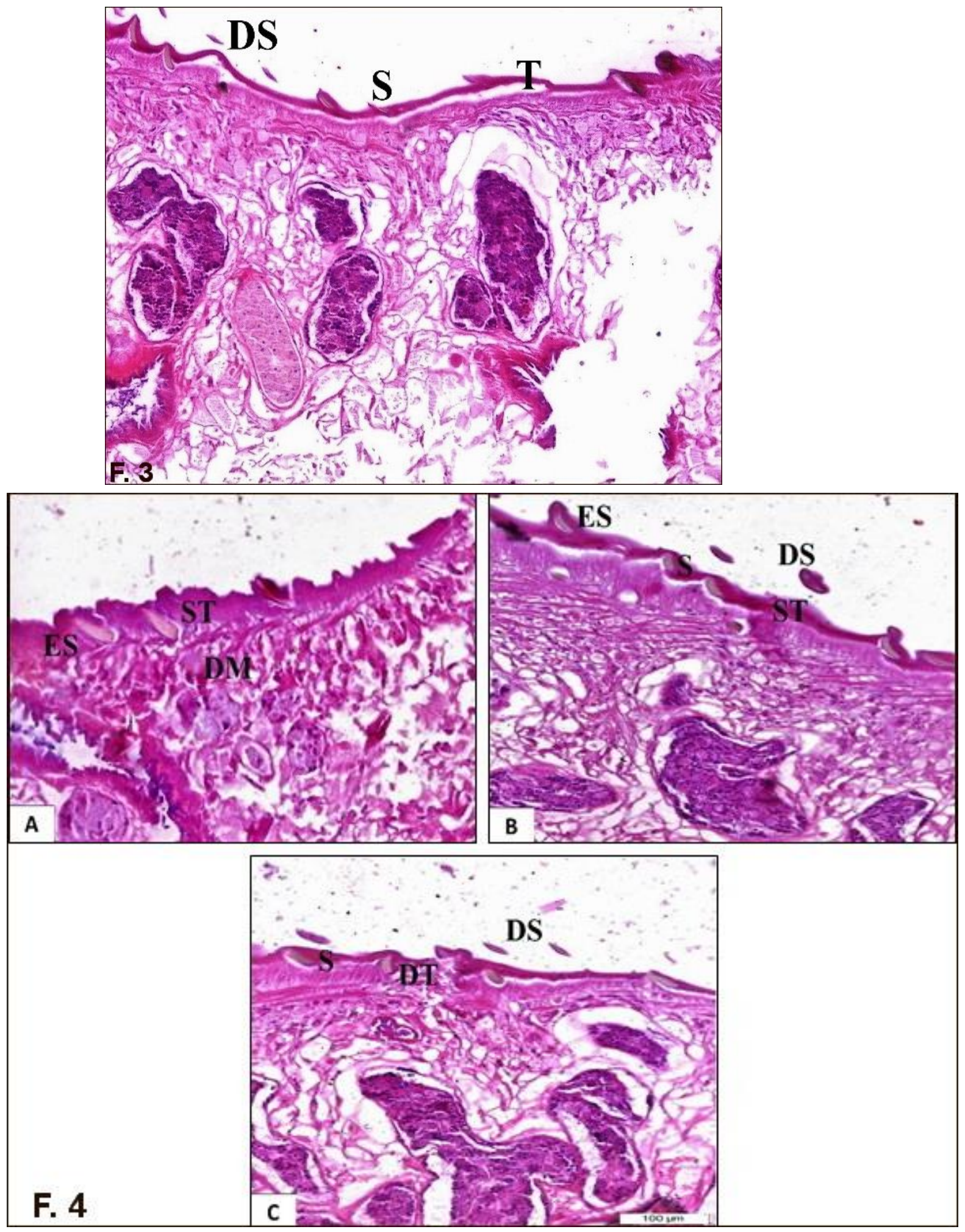


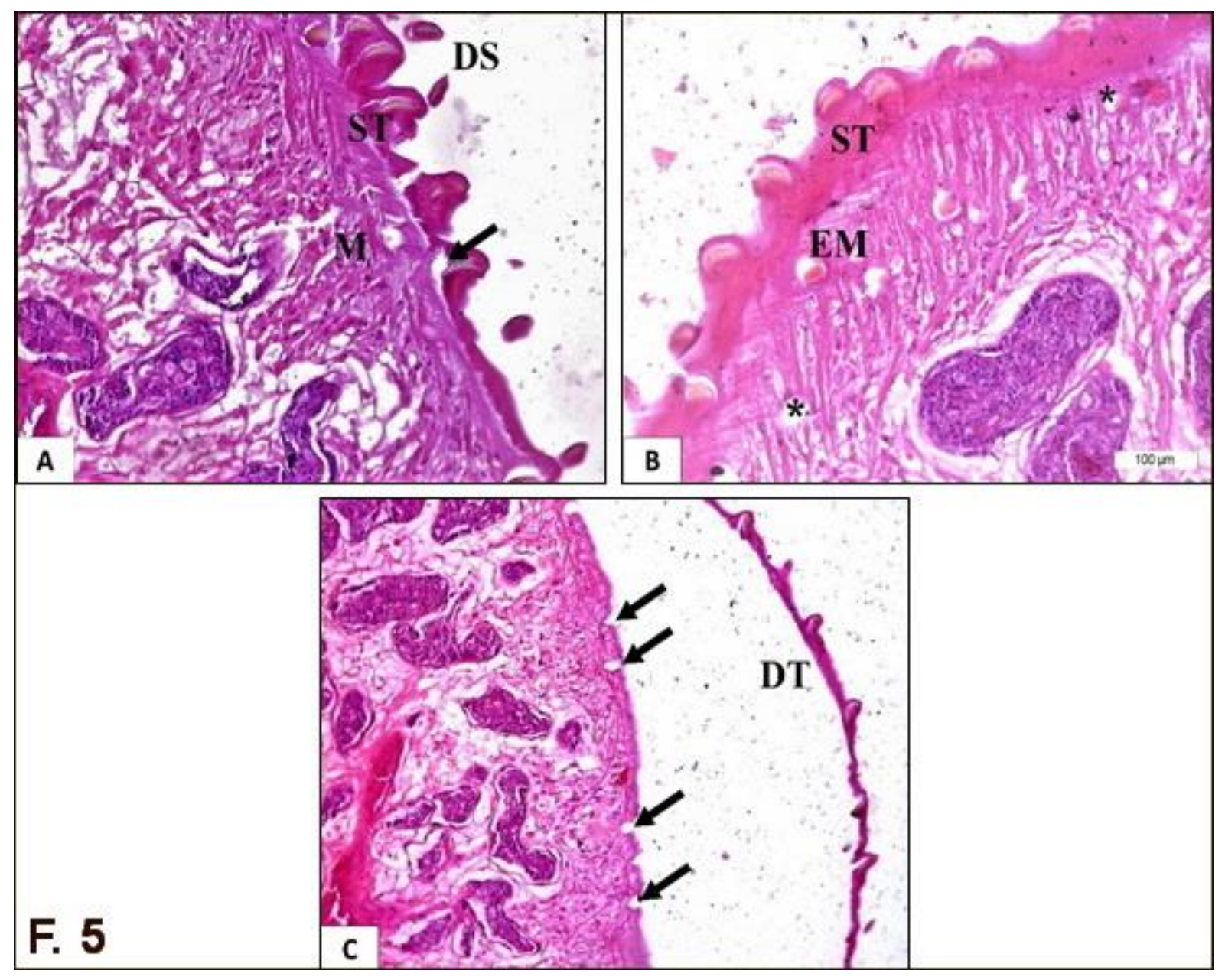

